\title{
Polymer properties to comply with requirements for medical devices
}

\author{
$\underline{\text { Joerg Vienken }}$ \\ Technical University Mittelhessen, Giessen. GERMANY, E-mail: vienken.usingen@gmail.com
}

\begin{abstract}
Polymers applied for medical devices need to be tested in depth before considering them suitable, during production of devices and in clinical application when being part of the final device. Both performance and stability, as well as blood compatibility, release of extractables and surface activity are keywords to be considered.
\end{abstract}

Keywords: Polymer performance, medical devices, blood compatibility, extractables, device approval

\section{Introduction}

Polymers used for the application in medical devices have to undergo harsh assessments and characterization. This involves in vitro and in vivo biocompatibility testing, qualification for purity. In addition, long-term stability of implanted medical devices under conditions of a possibly corrosive human environment is of paramount importance, whilst individual patient profiles, such as e.g., underlying diseases, like diabetes do further complicate polymer assessment and application.

\section{Physico-chemical stability}

Medical devices and composing polymers must show stability features already during production and sterilization processes. Thus, stability against acids, bases and solvents as well as the glass transition temperature in case of steam sterilization, are important properties.

\section{Blood compatibility}

Due to its peculiar composition, human and animal blood is able to wet any type of polymer, irrespective of its polymer formula. Based on its content of water, electrolytes, fat, carbohydrates and enzymes, blood allows for wetting the surface of both hydrophilic and hydrophobic, as well as the surface of polymers with domain structures. As a consequence, blood may provoke the release of extractables and induce subsequent adverse reactions. Examples for this notion are plasticizers and leachable members of the bis-phenol family. They show endocrine effects in patients. Thus, polymer purity or in case of polymer blends, careful action has to be taken in order to avoid or at least reduce leachable polymer compounds. A careful assessment of subsequent blood compatibility and stability by the bioengineer is recommended already in the early phases of device development.

\section{Reproducibility and performance}

Protein adsorption to material surfaces may interfere with long-term performance due to a secondary layer formation. E.g., fibrinogen adsorption may lead to platelet adhesion and clot formation. Functional groups, such as OH-groups, stimulate the plasmatic immune system (complement activation) and net negative charges on polymer surfaces may induce blood pressure drop.

\section{Summary}

Selection of polymers intended for the use in medical devices has to be precisely prepared and assessed. Surface properties of polymers need to be considered already in the early phase of development. Knowledge of polymer properties and on their stability under the condition of the human corrosive environment is a "must" for bioengineers. Under the current new Medical Device Regulations (MDR) in Europe, approving authorities, such as the European Medical Agency (EMA) in Amsterdam/NL, require polymer testing and characterization, both at the beginning of device development and during subsequent clinical use. 